\title{
Gender based EEG before and after acute bout of aerobic exercise
}

\author{
Nirmala Limbu', Ramanjan Sinha², Meenakshi Sinha², Bishnu Hari Paudel ${ }^{1}$ \\ ${ }^{1}$ Department of Basic \& Clinical Physiology, B.P. Koirala Institute of Health Sciences, Dharan, Nepal, ${ }^{2}$ Department of Physiology, All India \\ Institute of Medical Sciences, Raipur, India
}

Objective: We aimed to investigate how EEG frequency bands change in females in response to acute exercise compared to males. Methods: Consenting healthy adult females $(n=15) \&$ males $(n=15)$ bicycled an ergometer at 50\% HRmax for 20 min. EEG was recorded using 10-20 system from mid-frontal (F4 \& F3), central (C4 \& C3), parietal (P4 \& P3), temporal (T4 \& T3) \& occipital (O2 \& O1) regions. Exercise-induced EEG changes were compared between two sexes by Mann Whitney test. EEG power $\left(\mu \mathrm{V}^{2}\right)$ is presented as median \& interquartile range. Results: In females, as compared to males, resting right side delta, alpha, and beta activities were more in almost all recorded sites [delta: F4 = 49.82 (44.23-63.56) vs. 35.5 (32.70-44.44), p < 0.001; etc], [alpha F4: 127.62 (112.89-149.03) vs. 49.36 (46.37-52.98), $p<0.001$; etc], [beta F4 = 18.96 (15.83-25.38) vs. 14.77 (10.34-17.55), $\mathrm{p}<0.05 ; \mathrm{C} 4=21.16$ (18.4-25.9) vs. 15.48 (9.66-19.40), $\mathrm{p}<0.01$; etc]. Similarly, females resting right theta activity was more in parietal $[P 4=33.04(25.1-42.41)$ vs. 22.3 (18.36-34.33), $p<0.05] \&$ occipital $[02=50.81$ (30.64-66.8) vs. 26.85 (22.18-34.42), $\mathrm{p}<0.001]$ regions than in males. They had similar picture on the left side also. The delta values of right alpha power was less in female in frontal [F4 $=-11.61(-45.24-3.64)$ vs. $9.48(1.05-16.58), p<0.01]$ and central $[C 4=-72(-32.98-9.48)$ vs. 22.69 (13.03-33.05), $\mathrm{p}<0.01]$ regions compared to males. Also, they had less delta values of left central alpha $[\mathrm{C} 3=-8.32(-32.65-6.1)$ vs. $16.5(0.36-36.36), p<0.01]$ and temporal beta $[$ T3 $=-6.29$ (-10.09- -1.49) vs. 1.24 (-0.84- 2.8), p < 0.001] power compared to males. Conclusion: At rest females may have high EEG powers in different bands. In response to acute exercise, they respond in reverse way as compared to males.

Key words: Acute aerobic exercise, EEG, Females and Males

\section{INTRODUCTION}

Physical exercise is advocated because it maintains and enhances physical and mental healths. ${ }^{1,2}$ On mental well being, exercise is beneficial to mood and cognition, ${ }^{3,4}$ it reduces stress, decreases anxiety, and alleviates depression. ${ }^{5}$ All these subjective experiences in response to acute bouts of aerobic exercise are correlated with brain activity. Previous studies have shown that exercise is associated with changes in brain activity. ${ }^{6}$ These changes in brain activities are related with affective and perceptual responses to acute bouts of exercise. ${ }^{7,8}$ Electroencephalography (EEG) is one of the tools to measure the changes in the brain activity in response to exercise.
Access this article online

Website:

http://nepjol.info/index.php/AJMS

DOI: $10.3126 / a j m s . v 6 \mathrm{i} 2.11116$ 
bouts of exercise must first be compared. Therefore, we aimed to investigate how EEG frequency bands change in females in response to acute exercise compared to males.

\section{MATERIALS AND METHODS}

This comparative cross-sectional study was conducted on healthy adult ( $\mathrm{n}=30$; male $=15$; female $=15)$, with age ranging from 19 to 29 years in the Neurophysiology Laboratory, Department of Basic and Clinical Physiology, B.P. Koirala Institute of Health Sciences, Dharan, Nepal. In female, the EEG was recorded in the midluteal (20-24 days) phase of their menstrual cycle. Subjects with any cardio-respiratory problem/disease or physical disabilities likely to affect normal performance in the exercise stress test, suffering from seizure disorder and psychoactive substance use disorder, using or having any other drug/disorder that might affect EEG were excluded from the study. Prior to the recording, subjects were advised to come with a clean scalp without oil after two hours of light meal between 9 am to 11 am. Room temperature of the laboratory was maintained at $26 \pm 2^{\circ} \mathrm{C}$. The subjects were familiarized with the laboratory set up. The experimental protocol was explained and then informed written consent was taken from each subject. The EEG electrodes (Nihon Kohden) were placed at mid-frontal (F3-F4), temporal (T3-T4), central (C3-C4), parietal (P3-P4), and occipital (O1-O2) regions following the International 10-20 System of Electrode Placement. The reference electrodes were placed on the left and right earlobe (A1 \& A2). These electrodes were connected to the 16-channel digital EEG machine (Nihon KohdenNeurofax: Optiplex GXMT 5120). Impedance of electrodes was maintained at less than 5 kilohms. The probe of the pulse oxymeter (OLV-1100/1200 series, Nihon Kohden) was placed in the index finger to monitor heart rate (HR) and saturation of oxygen. The resting EEG was recorded in a supine position with eyes closed, relaxed condition. It was recorded till alpha waves were observed (more than $50 \%$ of the recording) for at least $10 \mathrm{~min}$. Then, the subjects started to pedal the automated bicycle ergometer (Model Ergometrics 900 ergoline D72475 Bitz, Germany) at a rate of $50 \mathrm{rpm}$ for male and $40 \mathrm{rpm}$ for female with the fixed EEG electrodes on the scalp and keeping oxymeter probe on the index finger. They exercised for $20 \mathrm{~min}$, maintaining the $50 \%$ of HRmax. To maintain it, the load was increased by 10 watts at every 2 min of exercise till a constant load was reached at 30 and 20 watts for male and female respectively. After exercise, again EEG was recorded in a supine position with eyes closed and recorded till HR returned to the baseline level.

The EEG records were inspected visually for artifacts. Then, artifact free six sec five epochs were selected randomly from each of the pre- and post-exercise sections of the record. Fast Fourier transformation (FFT) was performed on these data for power spectral analysis. The EEG power obtained from each epoch was averaged. Statistical analysis was done using SPSS (version 11.5). Exercise-induced EEG changes were compared between two sexes by Mann Whitney test. EEG power $\left(\mu V^{2}\right)$ is presented as median and interquartile range. A $P$ value of $<0.05$ was considered as statistically significant.

\section{RESULTS}

Age of the female $(21.93 \pm 4.09$ years) was comparable to the male $(24.87 \pm 2.75$ years $)$.

In female, as compared to male, resting (pre-exercise) right sided delta, alpha, and beta activities were more in almost all recorded sites [delta: F4 $=49.82$ (44.23-63.56) vs. 35.5 (32.70-44.44), $\mathrm{p}<0.001 ; \mathrm{C} 4=48.79$ (38.34-58.64) vs. $37.6(26.72-43.80), \mathrm{p}<0.01 ; \mathrm{P} 4=48.6$ (43.41-53.12) vs. 35.08 (29.48-38.98), $\mathrm{p}<0.001 ; \mathrm{O} 2=58.88(52.65-59.52)$ vs. $34.6(28.16-47.80), \mathrm{p}<0.001 ; \mathrm{T} 4=17.78(15.77-20.95)$ vs.14.13 (11.74-17.34), p <0.05], [alpha F4 = 127.62 (112.89-149.03) vs. 49.36 (46.37-52.98), p <0.001; C4: 124.95 (111.95-147.25) vs. 57.07 (51-59.29), $\mathrm{p}<0.001 ; \mathrm{P} 4=177.42(111.03-196.64)$ vs. 89.91 (66.58-98.86), $\mathrm{p}<0.001 ; \mathrm{O} 2=396.66(267.61-424.02)$ vs. $171.22(142.23-209.91, \mathrm{p}<0.001 ; \mathrm{T} 4=39.57$ (28.5-43.66) vs. 23.24 (17.56-24.32), p < 0.001], [beta $\mathrm{F} 4=18.96(15.83-25.38)$ vs. $14.77(10.34-17.55), \mathrm{p}<0.05$; $\mathrm{C} 4=21.16$ (18.4-25.9) vs. 15.48 (9.66-19.40), $\mathrm{p}<0.01$; $\mathrm{P} 4=22.88$ (18.97-32.84) vs. 18.7 (12.32-24.09), $\mathrm{p}<0.05$; $\mathrm{O} 2=47.84(28.08-66.29)$ vs. $27.86(21.80-35.03, \mathrm{p}<0.01]$ (Figure 1-a, 1-e, 1-g). Similarly, female resting right theta activity was more in parietal $[\mathrm{P} 4=33.04(25.1-42.41)$ vs. 22.3 (18.36-34.33), $\mathrm{p}<0.05]$ \& occipital [O2 $=50.81$ (30.64-66.8) vs. 26.85 (22.18-34.42), p <0.001] regions than in male (Figure 1-c). They had similar picture on the left side also (Figure 1-b, 1-d, 1-f, 1-h).

Immediately after $20 \mathrm{~min}$ of aerobic exercise on automated cycle ergometer, the delta values of EEG activity (difference between post exercise and resting) between female and male activity was compared. We found that the delta values of right alpha power were less in female in frontal and central regions compared to male (Table 1). Also, they had less left central alpha and left temporal beta power compared to male after the acute aerobic exercise (Table 2).

\section{DISCUSSION}

Exercise is associated with changes in brain activity, which is been measured by EEG. ${ }^{6}$ The gender-related brain 


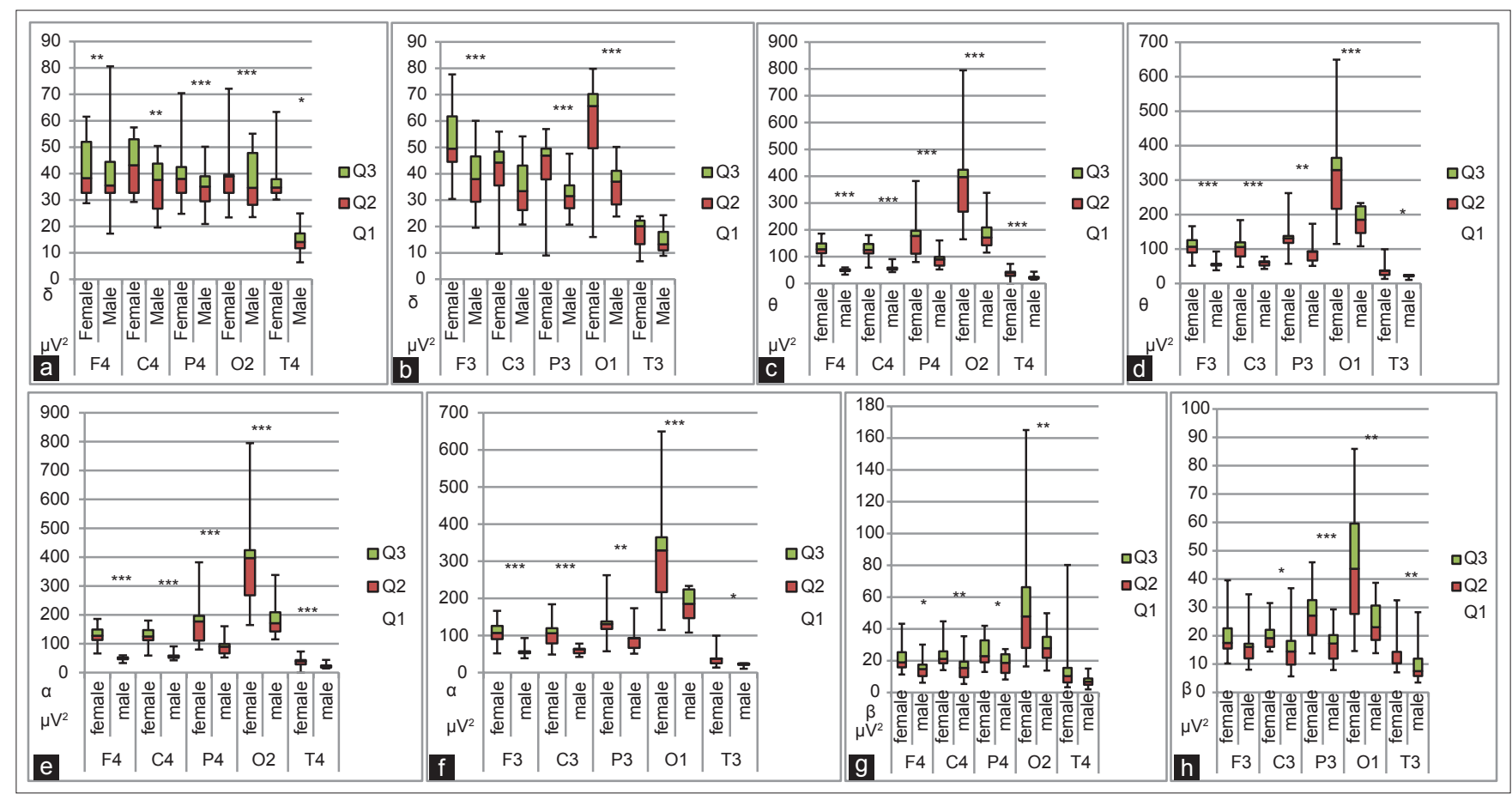

Figure 1: Comparison of EEG activity between female and male before exercise. (a) Pre-exercise right hemispheric delta activity between female and male. (b) Pre-exercise left hemispheric delta activity between female and male. (c) Pre-exercise right hemispheric theta activity between female and male. (d) Pre-exercise left hemispheric theta activity between female and male. (e) Pre-exercise right hemispheric alpha activity between male and female. (f) Pre-exercise left hemispheric alpha activity between female and male. (g) Pre-exercise right hemispheric beta activity between female and male. (h) Pre-exercise left hemispheric beta activity between female and male. $\left({ }^{*} \mathrm{P}<0.05,{ }^{* *} \mathrm{P}<0.01,{ }^{* * *} \mathrm{P}<0.001\right.$ )

\begin{tabular}{|c|c|c|c|c|c|c|c|c|}
\hline \multirow{2}{*}{$\begin{array}{l}\text { Delta value } \\
\text { Right EEG ( } \mu \text { V2) }\end{array}$} & & \multicolumn{3}{|c|}{ Female } & \multicolumn{3}{|c|}{ Male } & \multirow[t]{2}{*}{$P$ value } \\
\hline & & Median & Q1 & Q3 & Median & Q1 & Q3 & \\
\hline Alpha & $\begin{array}{l}\text { F4 } \\
\text { C4 }\end{array}$ & $\begin{array}{l}-11.61 \\
-7.20\end{array}$ & $\begin{array}{l}-45.24 \\
-32.98\end{array}$ & $\begin{array}{l}3.64 \\
9.48\end{array}$ & $\begin{array}{c}9.48 \\
22.69\end{array}$ & $\begin{array}{c}1.05 \\
13.03\end{array}$ & $\begin{array}{l}16.58 \\
33.05\end{array}$ & $\begin{array}{l}0.026 \\
0.005\end{array}$ \\
\hline
\end{tabular}

\begin{tabular}{|c|c|c|c|c|c|c|c|c|}
\hline \multirow{2}{*}{$\begin{array}{l}\text { Delta value } \\
\text { Left EEG }(\mu \mathrm{V} 2)\end{array}$} & & \multicolumn{3}{|c|}{ Female } & \multicolumn{3}{|c|}{ Male } & \multirow[t]{2}{*}{$P$ value } \\
\hline & & Median & Q1 & Q3 & Median & Q1 & Q3 & \\
\hline Alpha & C3 & -8.32 & -32.65 & 6.10 & 16.50 & 0.36 & 36.36 & 0.017 \\
\hline Beta & T3 & -6.29 & -10.09 & -1.49 & 1.24 & -0.84 & 2.80 & 0.0002 \\
\hline
\end{tabular}

differences are reflected in the electroencephalographic (EEG) activities. ${ }^{10}$ However, there are very few reports on exercise-induced changes in brain activity between the genders. We aimed to investigate how EEG frequency bands change in females in response to acute exercise compared to males.

We found that in females, as compared to males, in both (right \& left) hemispheres, resting EEG activity was more in all frequency bands at almost all recorded sites as in other study. ${ }^{11}$ The source of variation in EEG power between the genders could be anatomical variations, like, in the properties of the volume conduction system, particularly skull thickness. ${ }^{12} \mathrm{Li}$ et al reported that the cranium thickness is larger in females particularly frontal, parietal and occipital bones compared to males. ${ }^{13}$ There are reports stating significant negative association between skull thickness and electrophysiological parameters like EEG, Magnetoelectroencephalogram (MEG), eventrelated P300. ${ }^{14,15}$ However, Hagemann D et al observed only mediocre associations between the amplitude of resting EEG and skull thickness. On that observation, they concluded that it is the intracranial sources contribute much more variance to the surface EEG than do variations 
in skull thickness. ${ }^{12}$ Skull thickness may be neglected as a potent source of error when individual differences in brain activity are indexed by the magnitude of EEG alpha activity. ${ }^{12}$ Therefore, from the various intracranial sources, one of them could be hormonal effect on brain activity which generate variance of EEG powers between the genders.

In order to make uniformity, we have measured EEG in the luteal phase of the menstrual cycle in females. However, most of the studies, similar with ours, have not mentioned in which phase of the menstrual cycle they have recorded the EEG in the females. Early follicular phase have low level of estrogen and progesterone, ovulatory phase have high level of estrogen but low level of progesterone and luteal phase have high level of both progesterone and estrogen. ${ }^{16}$ Studies have shown that EEG activity changes with the phases of menstrual cycle in females. ${ }^{17,18}$

According to the phases of menstrual cycle alter cortical excitability in females. Estradiol enhances excitatory neurotransmission whereas progesterone inhibits it. ${ }^{19,20}$ During luteal phase, reports have shown that there is a tendency for increased alpha, and decreased beta power $\%$, reflecting the effect of progesterone. ${ }^{18}$ In a task-condition, like during thinking and mental relaxation, Krug $\mathrm{R}$ and his co-investigators found that influences of the menstrual phase on EEG activity were most obvious during mental relaxation. In that condition, they observed low alpha and theta activity at frontal and parietal regions during the luteal than ovulatory phase. ${ }^{17}$ Accordingly, the pattern of EEG activity induced by exercise must be also changes with the menstrual cycle, which should be considered in females.

Immediately after acute aerobic exercise, we observed less alpha activity in female as compared to male at right frontal and central (right and left) regions. Most studies have reported an increase of alpha activity during and following an acute bout of exercise. ${ }^{21,22}$ Increased alpha activity reflects a state of decreased cortical activation indication of fatigue, relaxation or decreased anxiety whereas decreased reflects cortical activation. ${ }^{23,24}$ Our observation showed that females are less relaxed or increased anxiety immediately after acute aerobic exercise as compared to males.

In addition, we found that immediately after acute exercise, in female, beta activity was less in left temporal region as compared to male. Previous studies studying the effect of acute aerobic exercise on brain activity have shown increase in beta activity. ${ }^{25-27}$ These authors suggest that increased beta activity after exercise is related to greater cortical activation. Although the underlying causes of exercise on alteration of EEG power are not clear, several speculations are made. Decrease in cerebral blood flow has shown decrease in beta (and alpha) activity. ${ }^{28}$ Increase in cerebral blood flow as a result of exercise is one of possibility to changes in EEG ${ }^{29}$ The altered brain activity after exercise is also related to an attentional demand and a higher arousal level, ${ }^{21}$ and hypothalamic modulation of increased metabolism and temperature. ${ }^{26}$ Exercise enhances cortical activation represented by increased beta activity ${ }^{28,30}$ which probably has effect on information processing and psychological variables like attention and arousal. ${ }^{31,32}$

However, all these studies are carried out in males. The difference in beta activity between male and female has been discussed by several papers in terms of gender related differences in cortical architecture, like, the orientation and connections of cortical pyramid cells, the number of cortical neuronal cells and the number of synapses. ${ }^{33-35}$

We observed decreased beta activity in left temporal region in females compared to males immediately after the aerobic exercise. In females, we have measured EEG in luteal phase. Becker et al have shown that the electrical activity of the brain changes in a parallel with changed hormone levels. ${ }^{36} \mathrm{~A}$ study have shown that the cortical arousal and orienting response attenuates during luteal phase. ${ }^{37}$ Even there is slight decrease in aerobic capacity during the luteal phase has been reported. ${ }^{38}$ There are very few studies on the effect of female hormones on exercise-related changes in female brain activity. Given the same degree of exercise (50\% HRmax), we found that the females cortical activation is less or less arouse in luteal phase of menstrual cycle compared to male, which might give gender-related differences in cognitive processing and affect associated with exercise.

We have recorded the exercise-related changes in EEG activity only in the luteal phase of the menstrual cycle. Therefore, future direction would be to record exerciseinduced EEG activity in all phases of menstrual cycle, and then compare each phase of cycle with males. Also, we can study changes in cortical activity with exercise mode and intensity as previous study has shown that these variables influence the brain activity. ${ }^{39}$

\section{CONCLUSION}

At rest, females had more power of all the EEG frequency bands than the males. After acute aerobic exercise, the females had decreased alpha activity in right frontocentral regions, and left alpha activity was reduced in central region and beta activity in temporal region compared to males. These reverse response of females compared to males could be due to the effect of ovarian hormones of menstrual cycle in brain activity. In females, estrogen 
enhances cortical excitability and progesterone inhibits it. ${ }^{18,20}$ Attenuation of cortical arousal and orienting response as well as a slight decrease in aerobic capacity has been reported during the luteal phase. ${ }^{37,38}$ Increased alpha activity indicates decreased cortical activation like relaxation, fatigue or decreased anxiety. ${ }^{24,25}$ Increased beta activity after exercise is related to greater cortical activation. ${ }^{26-28}$ Both alpha activity, in right frontal, bilateral central regions, and beta activity, in temporal regions were decreased in females after exercise compared to males indicating that females seem less relaxed and their cortical activation is less than the males immediately after acute aerobic exercise during luteal phase of the menstrual cycle.

\section{REFERENCES}

1. Biswalter J, Collardeai $M$ and Rene A. Effects of acute physical exercise characteristics on cognitive performance. Sports Med 2002; 32(9):555-566.

2. Guszkowska M. Effects of exercise on anxiety, depression and mood. Psychiatr Pol 2004 Jul-Aug; 38(4):611-620.

3. Colcombe $S$ and Kramer AF. Fitness effects on the cognitive function of older adults: A meta-analytic study. Psychol Sci 2003 Mar; 14(2):125-130.

4. Tomporowski PD. Effects of acute bouts of exercise on cognition. Acta Psychol (Amst) 2003 Mar; 112(3):297-324.

5. Salmon P. Effects of physical exercise on anxiety, depression, and sensitivity to stress: A unifying theory. Clin Psychol Rev 2001 Feb; 21(1):33-61.

6. Helena M, Camila F, Andréa D, Mauricio C, Fernando P, Pedro R, et al. Beta and alpha electroencephalographic activity changes after acute exercise. Arq Neuropsiquiatr 2007; 65(3-A):637-641.

7. Nybo $L$ and Nielsen $B$. Hyperthermia and central fatigue during prolonged exercise in humans. J Appl Physiol (1985). 2001 Sep; 91(3):1055-1060.

8. Petruzzello $S \mathrm{~J}$, Ekkekakis $P$ and Hall, EE. Physical activity and affect: EEG studies. In: Acevedo E O Ekkekakis P eds. Psychobiology of Physical Activity. Champaign, IL: Human Kinetics, 2006, pp 111-128.

9. Swaab D F. Sexual differentiation of the brain and behavior. Best Practice \& Research: Clinical Endocrinology \& Metabolism 2007; 21(3):431-444.

10. Matsuura M, Yamamoto K, Fukuzawa $H$, Okubo $Y$, Uesugi $H$, Moriiwa $M$, et al. Age Development and Sex Differences of Various EEG Elements in Healthy Children and Adults Quantification by a Computerized Wave form Recognition Method. Electroencephalogr Clin Neurophysiol 1985 May; 60(5):394-406.

11. Wada $Y$, Takizawa $Y$, Jiang $Z Y$ and Yamaquchi N. Gender differences in quantitative EEG at rest \& during photic stimulation in normal young adults. Clin Electroencephalogr 1994 Apr; 25(2):81-85.

12. Hagemann D, Hewig J, Walter C and Naumann E. Skull Thickness and Magnitude of EEG Alpha Activity. Clinical Neurophysiology 2008; 119(6):1271-1280.

13. Li H, Ruan J, Xie Z, Wang $\mathrm{H}$ and Liu W. Investigation of the Critical Geometric Characteristics of Living Human Skulls Utilizing Medical Image Analysis Techniques. International Journal of Vehicle Safety 2007; 2(4):345-367.

14. Cuffin BN. Effects of local variations in skull and scalp thickness on EEG's and MEG's. IEEE Trans Biomed Eng 1993 Jan; $40(1): 42-48$.
15. Frodl T, Meisenzahl EM, Müller D, Leinsinger G, Juckel G, Hahn K, et al. The effect of the skull on event-related P300. Clin Neurophysiol 2001 Sep; 112(9):1773-1776.

16. Guapo UG, Graeff FG, Zani AC, Labate CM, dos Reis RM and Del-Ben CM. Effects of sex hormonal levels and phases of the menstrual cycle in the processing of emotional faces. Psychoneuroendocrinology 2009 Aug; 34(7):1087-1094.

17. Krug R, Mölle M, Fehm $H \mathrm{~L}$ and Born J. Variations across the menstrual cycle in EEG activity during thinking and mental relaxation. Journal of Psychophysiology 1999; 13(3):163-172.

18. Kaneda $\mathrm{Y}$, Ikuta $T$, Nakayama $\mathrm{H}$, Kagawa $\mathrm{K}$ and Furuta $\mathrm{N}$. Visual evoked potential and electroencephalogram of healthy females during the menstrual cycle. J Med Invest 1997 Aug; 44(1-2):41-46.

19. Smith MJ, Adams LF, Schmidt PJ, Rubinow DR and Wassermann EM. Effects of ovarian hormones on human cortical excitability. Ann Neurol 2002 May; 51(5):599-603.

20. Inghilleri $M$, Conte $A$, Currà $A$, Frasca $V$, Lorenzano $C$ and Berardelli A. Ovarian hormones and cortical excitability. An rTMS study in humans. Clin Neurophysiol 2004 May; 115(5):1063-1068.

21. Petruzzello SJ and Landers DM. State anxiety reduction and exercise: Does hemispheric activation reflect such changes? Med Sci Sports Exerc 1994 Aug; 26(8):1028-1035.

22. Youngstedt SD, Dishman RK, Cureton KJ and Peacock L J. Does body temperature mediate anxiolytic effects of acute exercise? J Appl Physiol 1993 Feb; 74(2):825-831.

23. Petruzzello SJ, Landers DM, Hatfield BD, Kubitz KA and Salazar W. A meta-analysis on anxiety-reducing effects of acute and chronic exercise: Outcomes and mechanisms. Sports Med 1991 Mar; 11(3):143-182.

24. Boutcher SH and Landers DM. The effects of vigorous exercise on anxiety, heart rate and alpha activity of runners and nonrunners. Psychophysiology 1988;25:696-702.

25. Nielsen $B$ and Nybo L. Cerebral changes during exercise in the heat. Sports Med 2003; 33:1-11.

26. Youngstedt S, Dishman R, Cureton K and Peacock L. Does body temperature mediate anxiolytic effects of acute exercise? J Appl Physiol 1993; 74:825-831.

27. Doyle LM, Yarrow K and Brown P. Lateralization of event-related beta desynchronization in the EEG during pre-cued reaction times tasks. Clin Neurophysiol 2005; 116:1879-1888.

28. Kraaier V, Huffelen AC, Wieneke GH, Worp V and Bär PR. Quantitative EEG changes due to cerebral vasoconstriction. Indomethacin versus hyperventilation-induced reduction in cerebral blood flow in normal subjects. Electroencephalography Clin Neurophysiol 1992; 82:208-212.

29. Lardon $M$ and Polich J. EEG changes from long-term physical exercise. Biol Psychol 1996; 44:19-30.

30. Kubitz $\mathrm{K}$ and Mott $\mathrm{A}$. EEG power spectral densities during and after cycle ergometer exercise. Res Q Exerc Sport 1996; 67:91-96.

31. Tomporowski $\mathrm{P}$ and Ellis N. Effects of exercise on cognitive process: a review. Psychol Bull 1986; 99:338-346.

32. Kamijo K, Nishihira Y, Hatta A, Kaneda T, Kida T, Hiqashiura T, et al. Changes in arousal level by differential exercise intensity. Clin Neurophysiol 2004; 115:2693-2698.

33. Sowell ER, Peterson BS, Kan E, Woods RP, Yoshii J, Bansal R, et al. Sex Differences in Cortical Thickness Mapped in 176 Healthy Individuals between 7 and 87 Years of Age. Cerebral Cortex 2007 Jul; 17(7):1550-1560.

34. Luders E, Narr K, Thompson P, Rex D, Woods R, Deluca H, et al. Gender Effects on Cortical Thickness and the Influence of Scaling. Human Brain Mapping 2006; 27(4):314-324. 
35. Alonso-Nanclares L, Gonzalez-Soriano J, Rodriguez J R and Felipe J De. Gender Differences in Human Cortical Synaptic Density. Proc Nati Acad Sci USA 2008 Sep 23; 105 (38):1461514619.

36. Becker D, Creutzfeldt OD, Schwibbe M and Wuttke W. Changes in physiological, EEG and psychological parameters in women during the spontaneous menstrual cycle and following oral contraceptives. Psychoneuroendocrinology 1982; 7(1):75-90.
37. Walpurger V, Pietrowsky R, Kirschbaum C and Wolf OT. Effects of the menstrual cycle on auditory event-related potentials. Horm Behav 2004 Dec; 46(5):600-606.

38. Lebrun CM. Effect of the different phases of the menstrual cycle and oral contraceptives on athletic performance. Sports Med 1993 Dec;16(6):400-430.

39. Brümmer V, Schneider $\mathrm{S}$, Abel T, Vogt $\mathrm{T}$ and Strüder HK. Brain cortical activity is influenced by exercise mode and intensity. Med Sci Sports Exerc 2011 Oct; 43(10):1863-1872.

\section{Authors Contribution:}

$\mathbf{N L}$ - designed the study, recorded the data, analyzed the data, drafted the manuscript and reviewed the manuscript; RS - Contributed to the study design and analysis of data, reviewed the manuscript; MS - contributed to the study design and analysis of data, reviewed the manuscript; BH Paudel - contributed to the analysis of data, reviewed the manuscript.

Source of Support: Nil, Conflict of Interest: None declared. 